\title{
VII. On an approximate method of finding the forces acting in magnetic circuits
}

\section{Richard Threlfall M.A. \& Florence Martin}

To cite this article: Richard Threlfall M.A. \& Florence Martin (1894) VII. On an approximate method of finding the forces acting in magnetic circuits, Philosophical Magazine Series 5 , 38:230, 89-110, DOI: $10.1080 / 14786449408620604$

To link to this article: http://dx.doi.org/10.1080/14786449408620604

曲 Published online: 08 May 2009.

Submit your article to this journal $\sqsubset x$

Џ Article views: 2

Q View related articles $₫$ 
Approximate Method of calculating Magnetic Forces. $\quad 89$ glowing in the lamp wherein the phosphorus does not burn, in the lamp with carbon dioxide the filament becomes only dull red, and in the others it remains obscure, but still the phosphorus burns there after a minute.

Though the thermal conductive power interferes, I believe convection to play an important part here, and I venture to predict that, if the incandescent lamp, whilst glowing, could be intensely cooled, by being immersed in liquid oxygen or liquid air, that Prof. Dewar has so readily at hand, it would at once attain a higher illuminating power*; as convection would still more be lessened, the remaining particles of air and mercury vapour would have their motion almost entirely exhausted. And hence it may be concluded that such a lamp would absorb less electric energy for emitting the same amount of liglit, when the degree of rarefuction is made the highest possible.

The Hagne, April 1894.

VII. On an Approximate Method of finding the Fories acting in Magnetic Circuits. By Richard Threlfald, M.A., Professor of Physics, University of Sydney; assisted by Florence Martin, Student in the University of Sydney †.

DURING the last three years I have had occasion to design a good many reciprocating electromagnetic mechanisms, and have frequently felt the want of some simple method of making the necessary approximate calculations of magnetic forces.

I have obtained very little satisfaction from the attempts I have made to calculate tractions, proceeding by the method of finding poles and applying the law of inverse squares.

This ill success led me to investigate the applicability of the methods established by Maxwell in the chapter "On Energy and Stress in the Magnetic Field" ("Electricity and Magnetism,' vol. ii. $\$ \S 641-644)$, with the following results.

$\S 1$. Theoretical Considerations.

The problem for solution in its simplest form is as follows:"Given an iron anchor-ring uniformly wound and interrupted at one point by an air-gap of any given dimensions-

* I tried this effect with a mixture of solid carbon dioxide and ether on an incandescent lamp, but the globe becomes covered with frozen aqueous vapour and the mixture itself is a hindrance to judging the brightness acquired; a transparent cold liquid therefore is far more suitable.

† Communicated by the Author. 
to calculate the forces tending to draw the ends of the iron ring together when the strength of current flowing in the magnetizing circuit and the duta of winding are given.

\$2. The position established by Maxwell is as follows:-

(1) The laws of magnetic force are such that magnetic forces may be regarded as the expression of a state of stress in the magnetic medium.

(2) The medium is stable under such a distribution of stresses.

(3) A series of expressions may be found for the stresses at any point in the magnetic field.

$\S 3$. Maxwell's investigation does not explicitly include the case of a body with inconstant permeability; but I cannot find that this in any way vitiates the argument. Professor J. J. Thomson shows ("Applications of Dynamics to Physics and Chemistry,' § 33) that Maxwell's results may be considered as being derived from the existence of a term $\frac{1}{8 \pi} \mathrm{HB}$ in the Lagrangian function for unit volume of a magnetic field. If the permeability is a function of the induction, however, in any part of the field, the more general expression $\frac{1}{8 \pi} \int \mathrm{H} d \mathrm{~B}$ must be substituted for the above and the results modified accordingly. I have not succeeded in doing this. It appears, therefore, that Maxwell's system as applied to iron does not cover all the ground, because a modification must be introduced on account of the inconstancy of the permeability, and also on account of the Villari effect as shown by Professor Thomson. There may also be other undiscovered additions to make.

\$ 4. A great step is necessary to pass from Maxwell's position-that magnetic forces may be regarded as the expression of stresses in the field-to the position that magnetic forces are such an expression. There is all the difference that exists between a theory and a fact.

Everything, however, tends to show that the fact is that the theory is probably true so far as it goes, and we will therefore provisionally adopt it, and see first what additional hypotheses are necessary. It is obvious at once that the stresses are "stresses in a medium," while the forces are mechanical forces acting on matter. We must therefore consider that the medium is "attached" to matter so as to allow the stresses to appear as forces. Now the stresses in the medium depend on the nature of the matter which is permeated by the medium. Thus in the cut anchor-ring 
referred to above $(\S 1)$, the stresses in the medium in the airgap are not at all the same as the stresses in the medium in the iron. In our entire ignorance of the connexion existing between the medium and matter, it is not to me at all clear that, in calculating the magnetic forces tending to close the ring, we ought to consider the stresses in æther in air, and those alone. It is at all events conceivable that the nature of the connexion between the medium and the iron may be modified in some manner by the internal stresses in the iron. Also the ordinary laws of magnetic and electromagnetic action received their experimental demonstration at low inductions; and we have no right to say, without experimental evidence, that some terms not contemplated by Maxwell mignt not begin to produce effects on the stresses in air at high inductions. In the parallel case in iron such stresses do in fact occur.

I therefore attribute great importance to the experimental verification of the results deduced from Maxwell's theory as applied to the traction between iron bars in general, and especially at high inductions.

$\$ 5$. Experimental position.-The simplest case is that of the traction between two plane faces of iron, the faces being either the terminals of otherwise closed iron circuits, or of very long bars.

The case of the ring has been implicitly investigated by Bidwell (Phil. Trans. 1886); and the case of short bars by Bosanquet explicitly (Phil. Mag. 1886, vol. xxii.). The latter is the only investigation I know of in which simultaneous observations of Induction and Tractive force were made. In both cases Maxwell's theory leads to the expression

$$
\mathrm{F}=\frac{\mathrm{B}^{2} \mathrm{~A}}{\mathrm{\gamma} \pi}
$$

for the force in air between two opposing plane faces of iron, infinitesimally separated, A being the area of the faces and $B$ the (uniform) induction-density.

The net result of Bosanquet's work was to show :-

(1) When B is below 5000 the Tractions observed are generally much too large.

(2) The formula does not hold when the air-gap is appreciable.

(3) It holds within about 5 per cent. up to very high inductions.

It is obvious, therefore, that there is room for more work on the subject.

$\S 6$. For the reasons given I felt very strongly that it was 
necessary to establish the truth, or rather the approximate exactness, of the theory in the simple case studied by Bosanquet before going on to apply it to other and more complicated cases.

Consequently I investigated the following matters:-

(1) Influence of length of bars.

(2) Influence of kind and size of pole-pieces.

(3) Influence of imperfections in the ballistic method.

(4) Cause of Bosanquet's failure to obtain agreement with theory at low inductions.

(5) Canse of similar failure (?) with an appreciable air-gap.

\$7. Method of Experimenting. -I wound a number of solenoids on brass tubes, placed the iron bars to be investigated axially in these solenoids, observing the usual precautions, and measured the force (by calibrated springbalances) requisite to pull the bars apart, the force being applied scrupulously parallel to the axis of the bars by means of links, pulleys, and strings. Great attention was paid to the state of the cut surfaces. I tried surfaces of all kinds.

(a) Merely filed by watchmaker's finishing files.

(b) Ground on flat whetstonos to a surface-plate.

(c) Scraped to a surface-plate.

(d) Ground by emery wheels.

(e) Turned flat-to a surface-plate. This takes a little skill.

(f) Optically ground by emery and diamond-dust and finished with putty-powder.

This requires a note. Of course the bars must be provided with shoes of many times their diameter to make the process a success, and these shoes must be of similar material to the bars. In order to save circumlocution I may state that both I and my assistant Mr. Cook are fairly expert at this kind of work, and we met with no real difficulty. The use of diamond-dust instead of emery saves a little time, but makes it more difficult to get a good result. I obtained two sets of bars with properly ground faces. One of these sets was of hard iron, and was not so good as Brashear's celebrated testplates, on account of a slight convexity on the part of one surface and a corresponding concavity on the part of the other. The other pair of bars were of soft Swedish iron well annealed; they were less than 1 centim. in diameter, and about 60 centim. long. The surfaces were as good as the test plates, i. e. perfect according to the present state of the art. I have little doubt that they are as good soft iron surfaces as have ever been prepared. This means that there was no inequality comparable with a wave-length of sodium-light on either surface. 
The bars were kept straight and aligned by well-fitting glass, or brass, or fibre tubes at the plane of contact. The fit was always just so good that no correction for friction was necessary. The magnetizing current was measured by a Siemens dynamometer, which was compared with suitable members of a chain of Kelvin balances. It was found that in this instrument the readings were correct within the limits of accuracy of reading. The current was supplied by storagecells.

The induction-coils were wound on brass bobbins with proper precautions. The bobbins were of different diameters, and were compared ballistically, and found to give identical results; hence it was concluded that they were all free from leakage errors.

The ballistic galvanometer was a fine instrument, specially made for this kind of work. It was calibrated by turning over a large coil which was splendidly wound, and which has been checked in many ways. I made use of the values for the vertical force obtained about nine months ago by Mr. Farr, from a long series of experiments in my laboratory under the best and most careful conditions, and with the Kew apparatus. In all cases the induction-throw on the galvanometer was checked by reversal of the galvanometer-corrections, except when experiment showed that nothing was gained by such reversals, the instrument being in another part of the laboritory to the magnetic system. In all cases large resistances from a box of coils, constructed and calibrated by myself from Cambridge standards, were inserted in the circuit so as to give the best range for the galvanometer.

\$ 8. Results of Experimenting.-The general result was that I got rather worse agreement than was noted by Bosanquet, especially at low inductions. I therefore set myself to find out the reason of this. I am ashamed to say how long it took me to clear up the difficulty. I investigated the following possible causes.

(1) Imperfection of galvanometer law. This was got over by adjusting resistances till the same deflexion was obtained both on turning over the earth-inductor and on magnetizing the iron.

(2) Effects due to residual state of the iron. These were got rid of by demagnetizing the iron by an alternator and slideresistance, and observing magnetizations with the current in both directions, and also on reversal. By comparing the three sets of deflexions I assured myself that the discrepancy was not due to any error of this kind. A similar procedure when taking the tractions led to a like result. 
(3) By using induction-coils of different diameters 1 assured myself that $I$ was really measuring the operative inductions.

(4) The state of the smrface of the bars is an important matter. If the ends are rough, of course the contact is at points. This leads to a concentration of induction at these places, and is a very constant source of error. My best bars, however, gave just as anomalous results as my ronghest ones, so that the deviation could not be attributed to this canse.

(5) Finally I tried, without much confidence, the effect of a small indirectness of pull. This was done by winding two wide solenoids and leaving sufficient space between their ends to see what was going on at the point of junction; a gasflame was put on the side of the junction, remote from the observer.

It was then found that one side of the bar which was being pulled off invariably remained in contact after the other side had slightly separated - when this was prevented by slightly guiding the spring-balance by hand, the agreement was as good as at higher inductions. The explanation is now obvious. If the bars separate slightly just at one side, two things happen. (1) The total reluctance of the circuit increases. (2) The induction concentrates at the parts in contact. At low inductions the effect of (2) overpowers that of (1). At high induetions, when the permeability of the iron becomes less, the induction is less free to distribute itself, and also the traction of the bars being greater, the phenomenon does not begin to manifest itself till rupture is just about to be produced, or it produces a very much smaller percentage error. Of course all this might have been foreseen, but one's experience with strong magnets-in which case it is notorious that it is much more difficult to pull off an armature straight than sidewaysmisled me.

On examining other bars which had given similar results at low inductions, I found I could similarly diminish or increase the apparent traction by varying very slightly the direction of pull. Thus with a pair of flat-ended bars and an induction of about 3000 C.G.S. the calculated pull was one pound seven ounces; but the observed pull was always over two pounds, and in some experiments about three pounds. On guiding the bars so that no wedge-shaped gap appeared, the traction could be got down to about one pound eight ounces. I do not think it is possible to get much closer than this, for if proper arrangements are made to absolutely ensure a really true and rigid separation, friction would inevitably come in 
to introduce errors. My results at higher inductions were so similar to Bosanquet's that they are not worth reproducing.

$\S 9$. With regard to the formula not applying with nonmagnetic gaps of sensible dimensions parallel to the lines of induction-as when Bosanquet separated the bars by wood and paper-the explanation is obvious. The lines of induction no longer leave the surfaces normally, and the conditions postulated by the formula are not in existence.

$\S 10$. Resulting position of the Theory.

When the bars are in contact, the stress theory, and what I will call the magnetic fluid theory, lead to the same result. Which is true certainly within about five per cent., and may be exactly true.

In any case, measuring tractions is not the way to get accuracy, though I have no doubt that rather better results could be got by going into the matter more elaborately than was done either by Bosanquet or myself.

In what follows I shall suppose that the theory is true, and that the real cause of magnetic forces is to be sought in some condition of the æther mechanism which receives a sufficient statistical definition from the Induction Diagram.

$\S 11$. The effect of varying the kind of iron employed should be the same as varying the induction-density - at least in so far as the phenomenon can be considered to depend on permeability. I used induction-densities of from 2000 to 18,000 , but could not detect any effect-when the cause of error referred to above was eliminated. I also used all kinds of iron, from annealed Swedish iron to ordinary cast iron. I varied the lengths of the bars from $60 \mathrm{~cm}$. to $6 \mathrm{~cm}$.- - and the diameter from about $2 \mathrm{~cm}$. to about $6 \mathrm{~cm}$.

In no case could I detect any deviation from the predicted traction which could not be explained by unavoidable experimental errors. With short bars and high inductions necessitating the use of very strong fields, some induction is included by the testing-coil which does not help the traction and which tends to make the calculated traction appear too large. When this source of error was eliminated, no greater discrepancies were observed with short bars than with long ones.

$\$ 12$. I conclude therefore

(1) The traction produced by a given tube of induction when running out of air into iron and crossing the surface normally is independent of the nature of the iron, or of its form. I had a difficulty in bringing myself to believe this, but the conclusion seems inevitable. 
Corollary (1): The magnetic forces are independent of the stresses in æther inside the iron.

Corollary (2). Setting aside Professor J. J. Thomson's stresses, the æther stress in air is less than that in ironassuming that Maxwell's "magnetic material" sufficiently represents iron. The difference of tensions is

or

$$
\begin{gathered}
\frac{\mathrm{B}^{2}}{8 \pi}-\frac{1}{4 \pi}\left(\mathrm{BH}-\frac{1}{2} \mathrm{H}^{2}\right), \\
\frac{(\mathrm{B}-\mathrm{H})^{2}}{8 \pi}-.
\end{gathered}
$$

This is an unbalanced stress; and if the lines of induction in the iron give rise to forces similar to those produced in air, this must mean that the boundary tends to be pulled off the iron. Taking Professor Thomson's stresses into account, this effect may easily be reversed in any actual case.

Referring to Professor Thomson's investigation (Physics and Chemistry), I cannot avoid the impression that there still remains a set of stresses depending on the variation of elastic constants with temperature. This would further complicato matters.

$\S 13$. Each tube of induction is therefore a tube of force within the usual definition; but it does not follow that the only forces are those represented by the tubes of induction. If the tubes leave the iron surface normally, then the pressural forces are tangential and we get the formula we have been using; and similarly if the tubes of induction are tangential (i.e. when the infinitesimal air-gap separates similar poles), the pressures operate alone, and we have a repulsion equal to the former attraction as in the elementary theory. If the tubes of induction leave the iron at any angle to the surface between 0 and $\pi / 2$, we must consider the effect of the pressural forces.

To calculate these effects it is convenient and perhaps correct to assume that just as the internal stresses of the iron do not affect the forces which are the expression of the external æther tensions. so they do not affect the forces corresponding to the hydrostatic pressures. If, therefore, we consider a line of force running out of iron into air and making an angle $\theta$ with the normal, we can estimate the direction and magnitude of the magnetic forces at once, thus :-

Let $A B$ be the trace of a plane boundary between air and iron, and $\mathrm{ON}$ a normal drawn outwards into air. Let $\mathrm{OP}$ be a vector in the plane of the paper representing the tensional 
force per unit area at a point about $O$. Draw $O Q$ perpendicular to $O P$ and in the plane of the paper. Then the pressural forces lie in a semicircle of which $O Q$ is a radius, and whose plane contains $O Q$. Since the pressures are symmetrical with respect to $O Q, O Q$ is their resultant, and

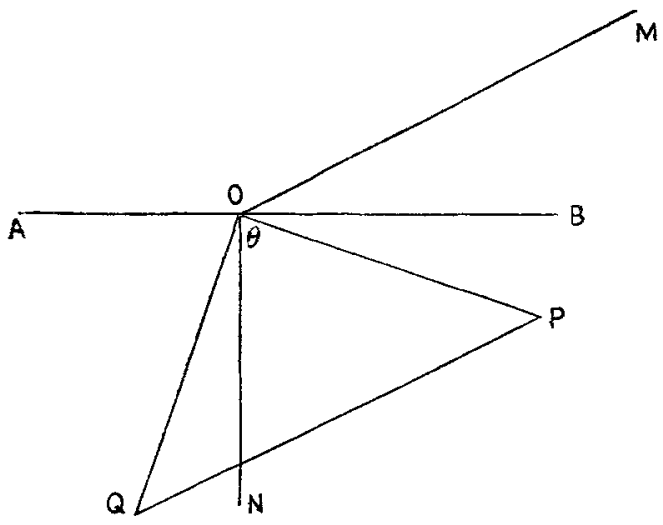

by the theory this is equal to $O P$-so that $O Q$ is the vector representing the pressures. A force represented by $\mathrm{OM}$ equal and parallel to $\mathrm{PQ}$ is therefore the resultant force, and clearly in this case is a repulsion whose magnitude along the normal produced is

$$
\mathrm{OP} \cdot \sqrt{2} \cos \left(\frac{3 \pi}{4}-\theta\right),
$$

an expression which gives no normal component at all when $\theta=\pi / 4$. The force is therefore an attraction or repulsion according as $\theta$ is less or greater than $\pi / 4$-and a shear at this point. I tried to observe this, but could not get the lines to leave the surface at the exact angle. However, the above way of looking at the matter is convenient when filings are used to trace the direction of the induction. This expression has been pointed out to me by Mr. Pollock as identical with that given by Maxwell's general formula in $\$ 643$ for the special case here considered.

It is now evident why it was that Bosanquet got results differing from those calculated from the formula for normal inductions, because, as filings show, a very small gap is sufficient to produce a marked spreading of the field.

$\$ 14$. By observing the distribution of filings about different air-gaps, it appeared probable to me that the following proposition might be true-as referring to bars of different

Phil. Mag. S. 5. Vol. 38. No. 230. July 1894. 
diameters:- "With similar pole-faces and the same permeability and induction-density, the induction (or filing) diagrams are similar when the length of the air-gap is the same fraction of a standard dimension of the pole-faces."

If this be true, it follows as a consequence that with similar air-gaps at similar induction-densities, the traction is the same fraction of the traction with the poles in contact, whatever be the actual dimensions of the poles.

The greater part of the experimental work I have to offer refers to this point, for, if established, we clearly have a method which will enormously facilitate the calculation of magnetic forces.

$\S 15$. The observations made on this subject are sufficiently detailed in the Tables I. to XIII., and the results will be understood by looking at the curve.

The tractions were measured by spring balances as before, measured pieces of brass being inserted between the pole-faces. In a series of observations the induction was kept constant by varying the magnetomotive force.

The observations were taken just as in the previous case. A little care is necessary in defining what is meant by the total induction. If the bars are long and thin, then of course the solenoidal condition is fulfilled pretty closely, and there is no ambiguity; but with large air or brass gaps--say, amounting to two diameters of the bars-the lines begin to leave the iron just in front of the middle point of each bar (at all events when the bars are about 50 diameters long). The "total induction" therefore has no very exact meaning" with respect to the iron, unless it be specified where it is to be measured.

At the time the experiments were made, I did not (as I now consider) sufficiently attend to this point, though I ased a testing-coil of about four times the diameters of the bars, and kept the coil just to one side of the gap when the latter was large. It is probable, therefore, that I have considerably overestimated the tractions with the larger air-gaps, for the induetion must have been greater than I took it to be. I have decided not to re-investigate this point, for the curve is of use in giving approximate ideas of tractions only; and no one, after looking at it, would design a mechanism with air-gaps as long as those which are probably inaccurate. I have made a little allowance for this (most unscientifically of course) in drawing the curve. In fact my suspicions were first aroused by examining the part of the curve corresponding to the larger air-gaps.

It will be seen that I examined a good many cases, and the 
results show that when the non-magnetic field is of sensible dimensions, the differences in the permeability of the samples examined do not lead to any very abnormal, results. . The curve is drawn by reduction for a bar one centimetre in diameter; and the air-gaps, which must be expressed in diameters, appear therefore in centimetres. The ordinates give the values of the tractions at corresponding points in terms of the calculated tractions when the surfaces are in contact.

One set of observations refers to square bars. In order to utilize the results, I assumed that the field would be distributed: very much as if the bar were round and of a diameter equal to the mean of the diameters of the inscribed and circumscribed circles.

To use the curve it is only necessary to express the length of the air-gap as a fraction of the diameter of the poleface, and refer to the table to find the proper factor to multiply the traction with the bars in contact at the proposed induction.

It will be seen that the factor is practically the same for all inductions-hence one curve only is given.

$\S 16$. In general it is more convenient to take the magnetomotive force as given; and in this case the indaction cannot be estimated without a knowledge of the reluctance of the circuit. Now methods of building up the characteristic curve of the magnet have been given when the air-gaps are narrow by Drs. J. and E. Hopkinson and others; but I thought that I might possibly be able to extend the method of similar. systems so as to include air-gap reluctances. In similar induction systems at constant induction-density, the reluctances of the gaps should be, roughly, inversely as the linear dimensions. I examined three sets of bars to see how near such an approximation really was, but it will be noticed that the results would not reduce so as to give a single curve by any such simple process. The curves are therefore kept separate ; they cover bars of from about one to three centim. in diameter. The induction was in these cases correctly measured at the centres of the bars. It was necessary to use the ampere-balances to get a sufficiently accurate knowledge of the magnetizing currents. The results are contained in Tables XIV. to XVI., and are also plotted for the mean of all inductions. The reluctance of the iron and air circuits was measured before the bars were cut and plotted against inductions. It was assumed that by using bars of the length employed, the air reluctance (other than that at the gap) would not be materially ehanged by pushing the bars up to H 2 
2 diameters apart. The proper reluctance for the iron and air circuits was taken from the curve in finding the reluctance of the air-gap.

Except with the largest bar, there is no definite indication of the reluctance depending on the induction-density. In this case separate curves might have been drawn, but I did not think it worth while to introduce a fresh sheet of curves.

I am not sure that a real reduction in air-gap reluctance at about 2 diameter has not been smoothed out; but as the observations are marked on the curves, any one will be able to form his own opinion.

It will be noticed that the curvature becomes very great when the air-gap amounts to about 1.5 diameter. It is, perhaps, not too much to say that the reluctance increases very fast as the gap increases to $\cdot 2$ diameter, after which it increases more slowly up to about 1 diameter, and then remains roughly constant.

\$17. I do not know whether a unit of reluctance has yet been adopted. It has been necessary for me to use one, however, and I take as unit reluetance that reluctance through which unit magnetomotive force produces unit induction. By unit magnetomotive force I mean that magnetomotive force whose C.G.S. value is unity-i.e., that produced by $\frac{1}{4 \pi}$ C.G.S. turns. If the permeability of air be taken as unity, then one cubic centimetre of air has unit reluctance on this system. There are, of course, other ways of defining unit reluctance ; but this is, I think; the only one that gets rid of the $4 \pi$ and leads to an easily realizable material standard.

$\$ 18$. The reluctance curves and traction curves are not unlike each other in general form, and enable us to draw some practically valuable conclusions as to the design of magnets intended to operate over air-gaps. For instance, with a given total induction the force at contact is inversely as the area, but the traction curve shows that this principle must not be pushed too far when we consider traction over an air-gap. Thus, I am told (though I do not believe it) that rock-drills will not work with a shorter stroke than five inches; the traction curve shows at once that for a given induction (a case which does not practically occur in every instance) it is possible to make the pole-pieces too small, if we wish to get the maximum work done during the stroke. This is independent of considerations arising when magnetomotive force, and not induction, is given. 


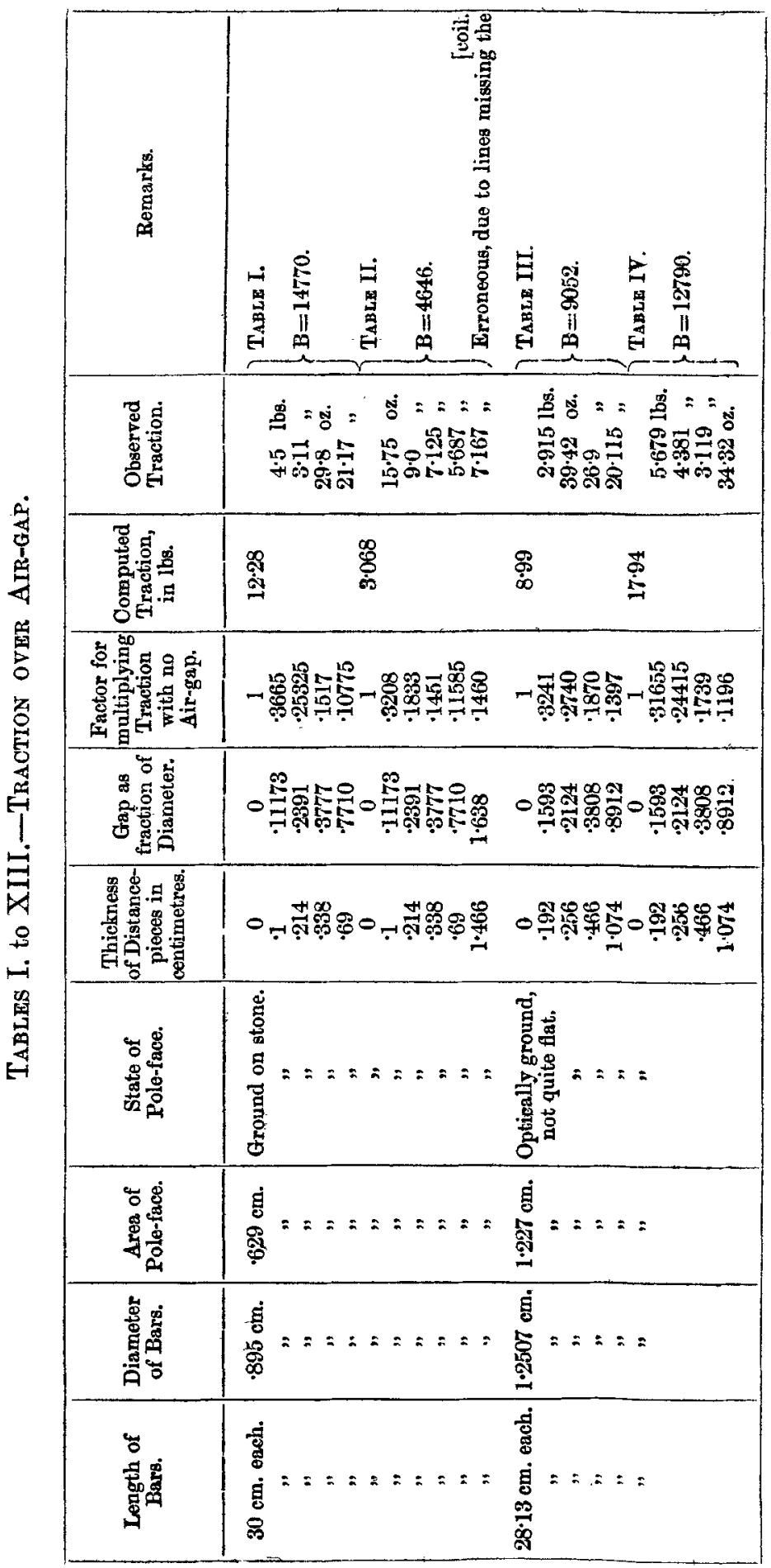




\begin{tabular}{|c|c|}
\hline 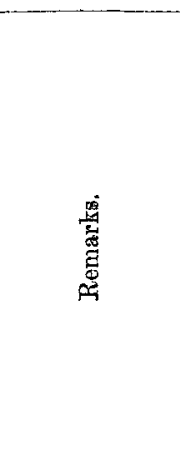 & 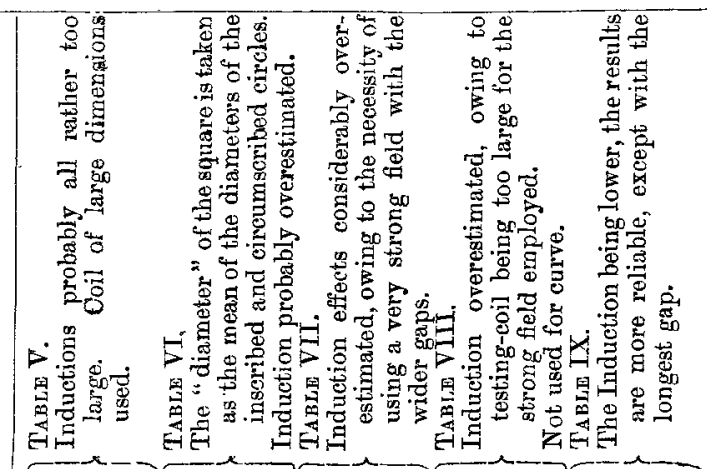 \\
\hline 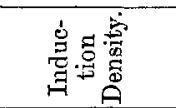 & 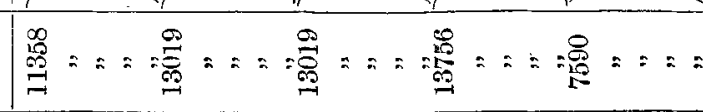 \\
\hline 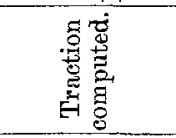 & 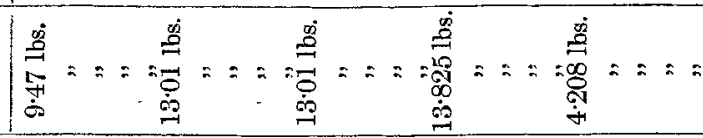 \\
\hline 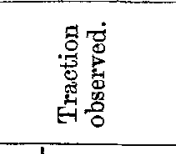 & 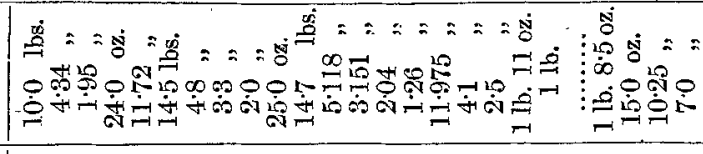 \\
\hline 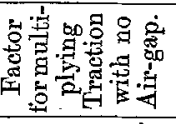 & 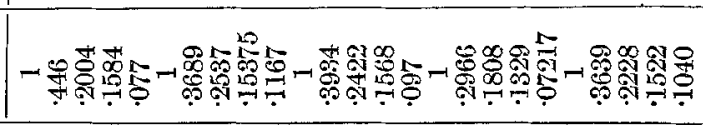 \\
\hline 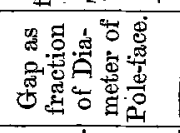 & 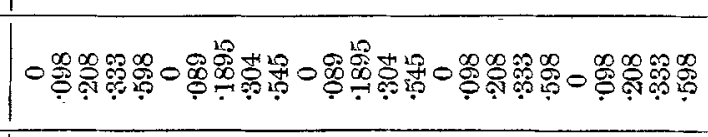 \\
\hline 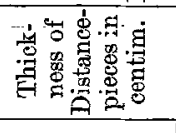 & o \\
\hline 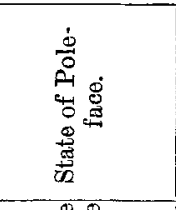 & 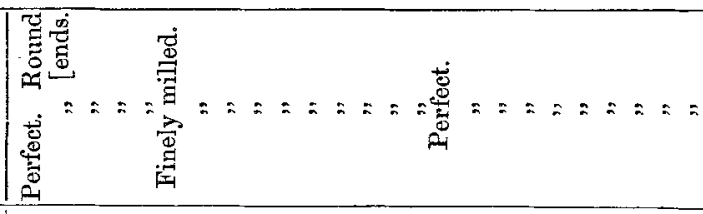 \\
\hline 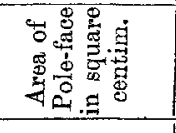 & 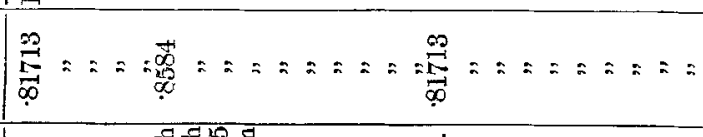 \\
\hline 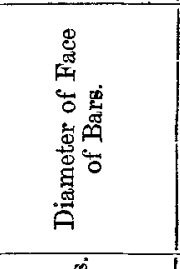 & 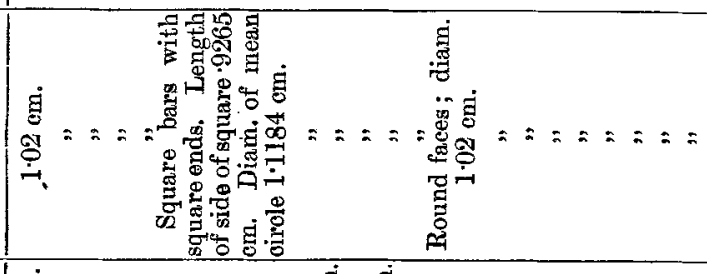 \\
\hline 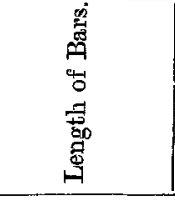 & 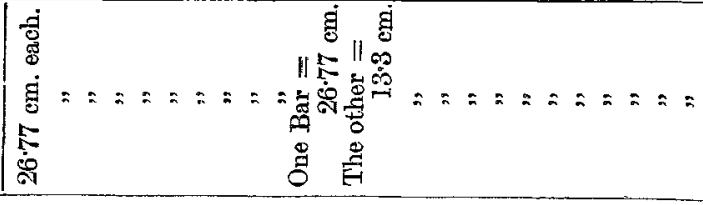 \\
\hline
\end{tabular}




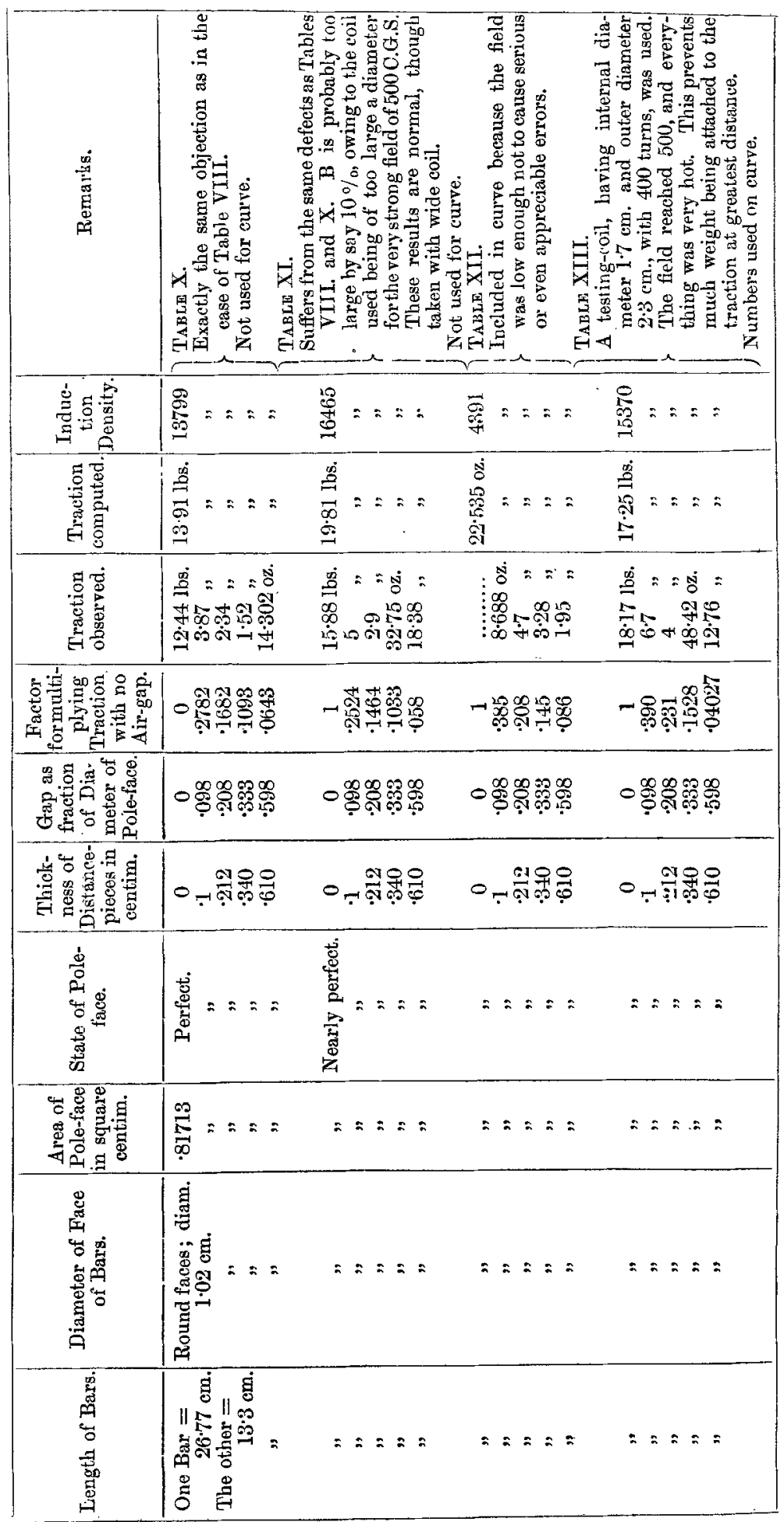




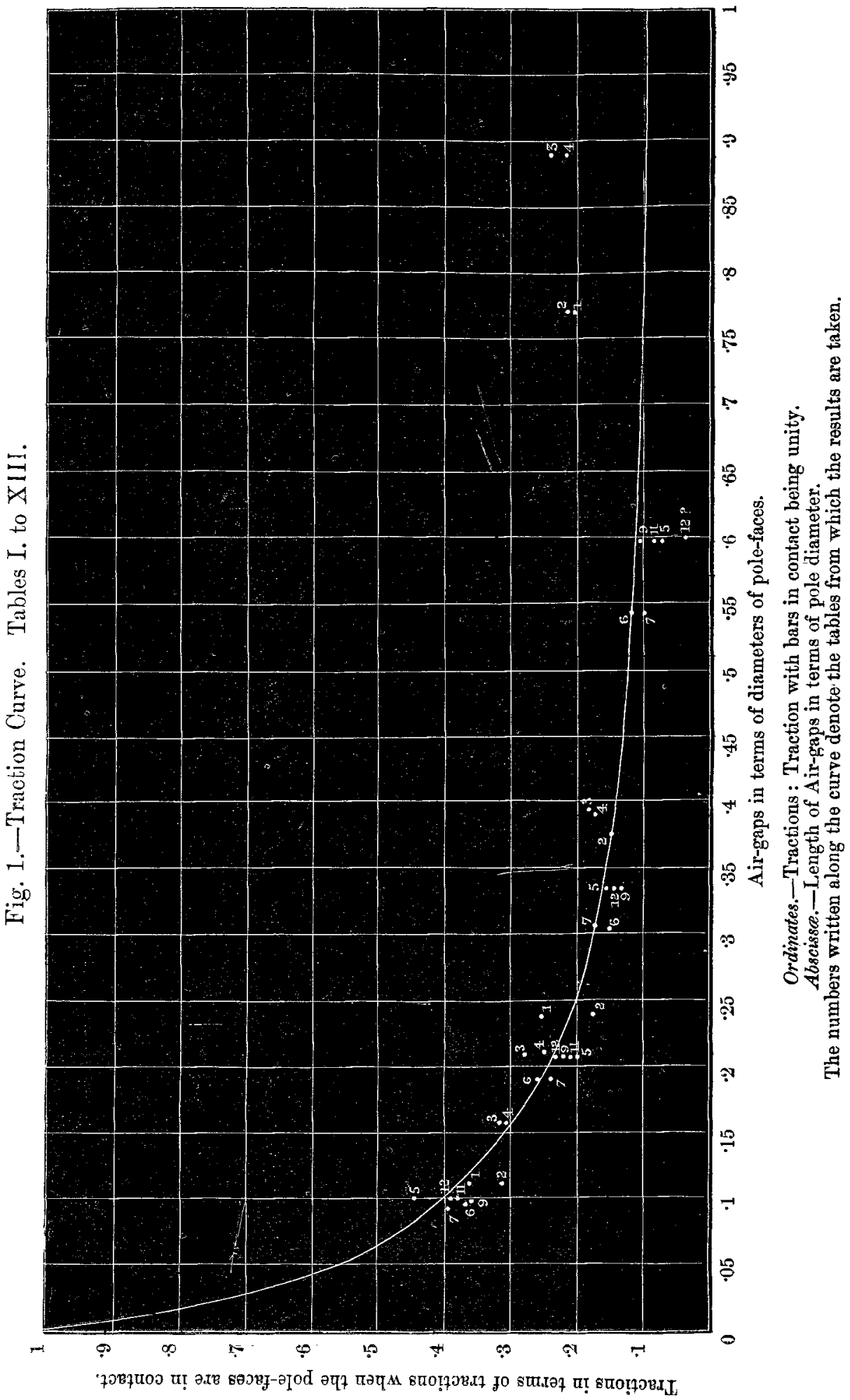


Approximate Method of calculating Magnetic Forces. 105

\begin{tabular}{|c|c|}
\hline 蛋 & 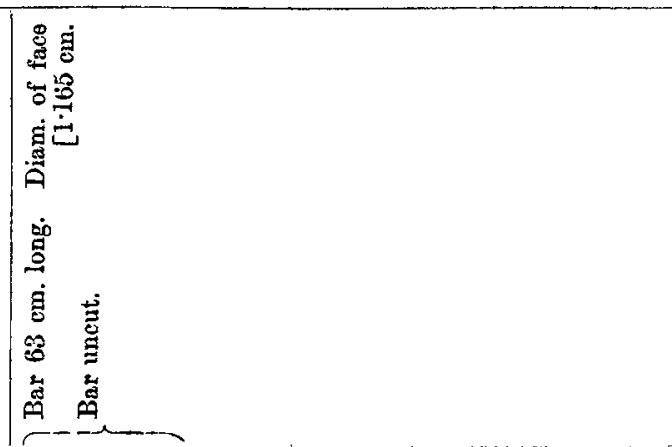 \\
\hline 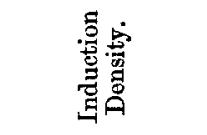 & 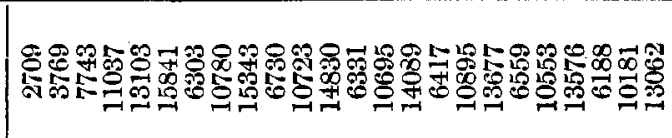 \\
\hline 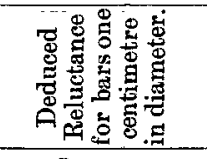 & 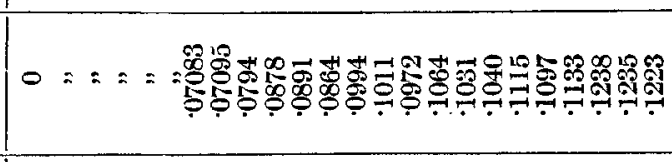 \\
\hline 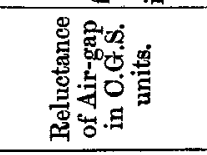 & 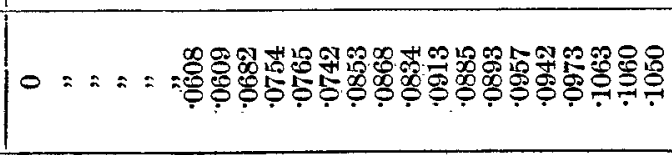 \\
\hline 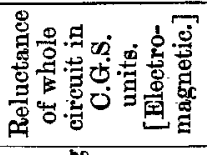 & 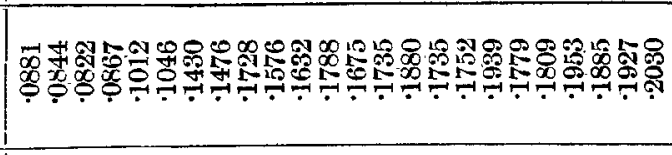 \\
\hline 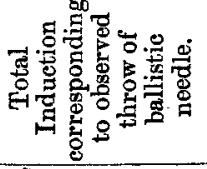 & 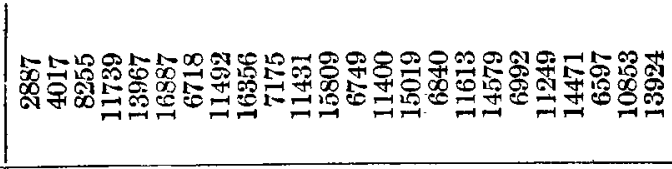 \\
\hline 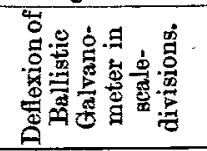 & 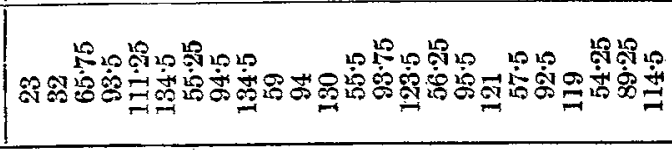 \\
\hline 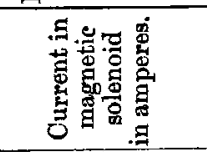 & 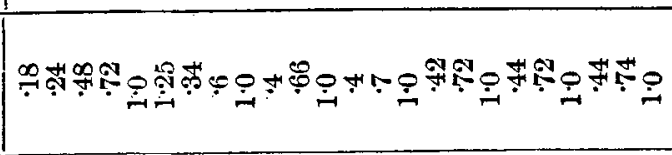 \\
\hline 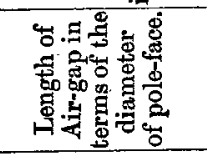 & 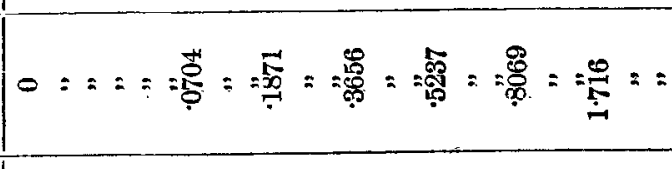 \\
\hline 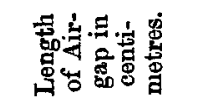 & | \\
\hline
\end{tabular}


106 Prof. R. Threlfall on an Approximate Method

\begin{tabular}{|c|c|}
\hline 离 & 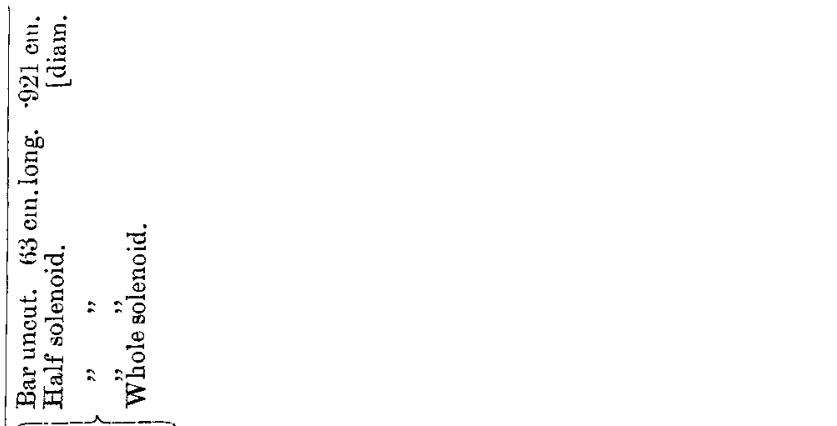 \\
\hline 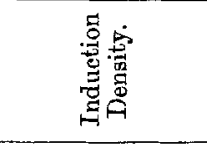 & 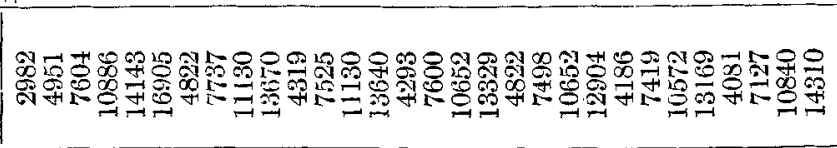 \\
\hline 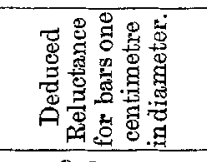 & 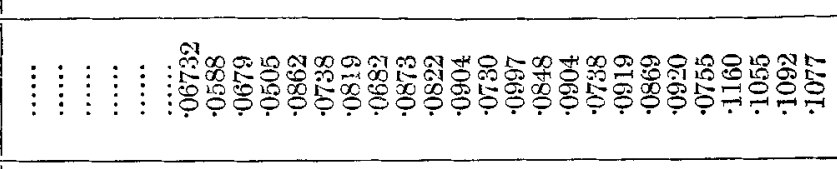 \\
\hline 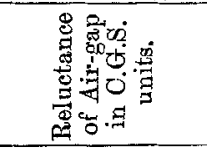 & 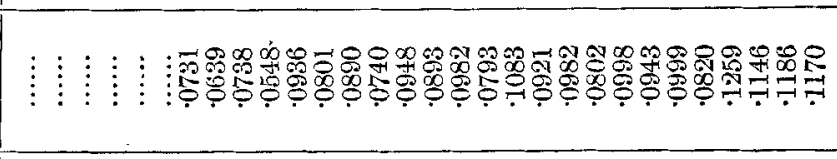 \\
\hline 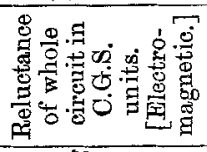 & 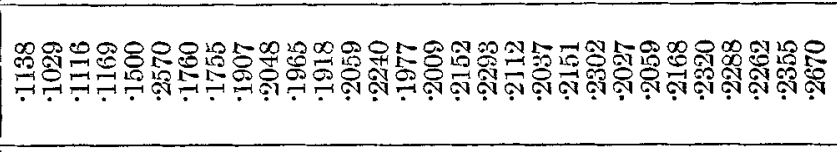 \\
\hline 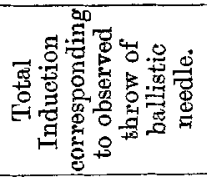 & 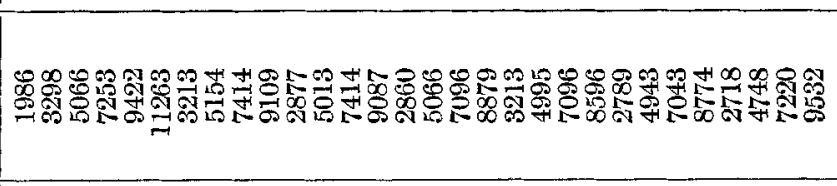 \\
\hline 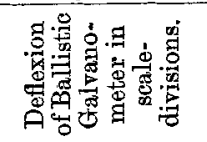 & 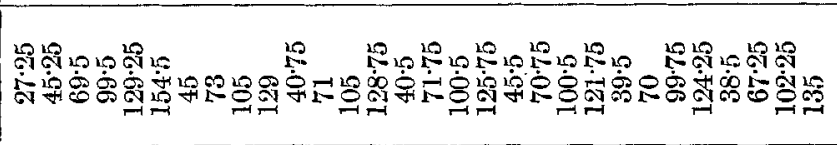 \\
\hline 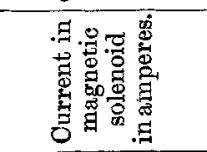 & 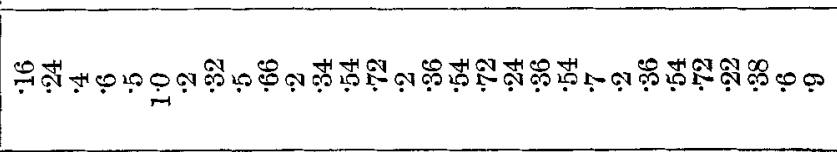 \\
\hline 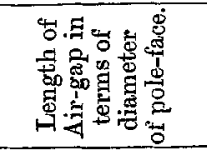 & $0=2==$ = \\
\hline 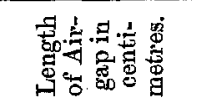 & 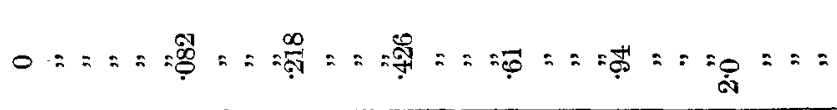 \\
\hline
\end{tabular}


of finding the Forces aeting in Magnetic Circuits. 107

\begin{tabular}{|c|c|}
\hline 离 & 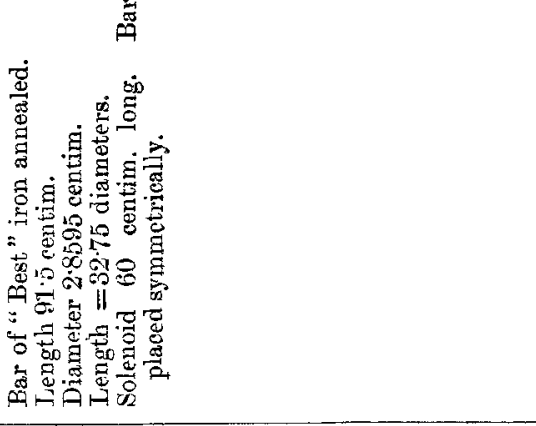 \\
\hline 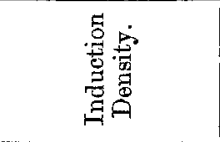 & 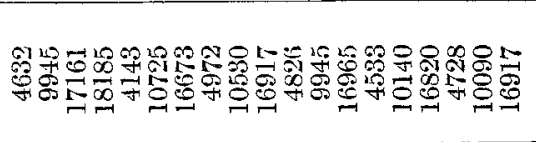 \\
\hline 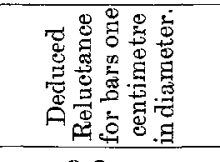 & $\vdots \vdots \vdots: \log ^{\circ}$ \\
\hline 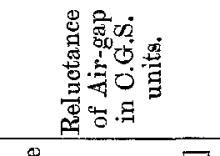 & 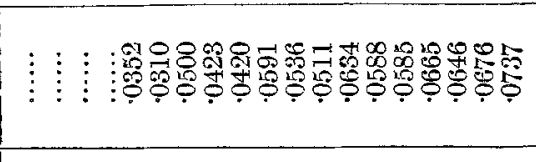 \\
\hline 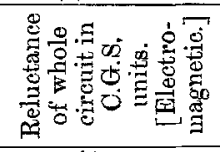 & 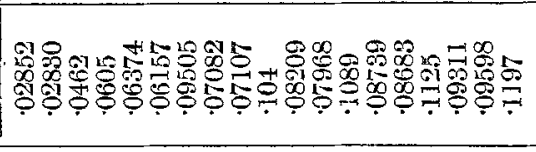 \\
\hline 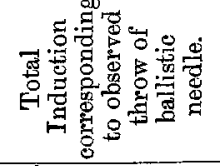 & 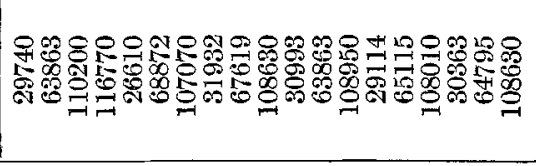 \\
\hline 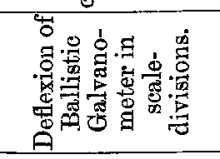 & 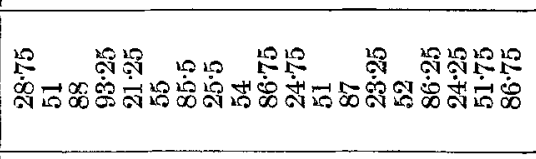 \\
\hline 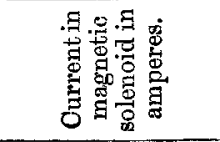 & 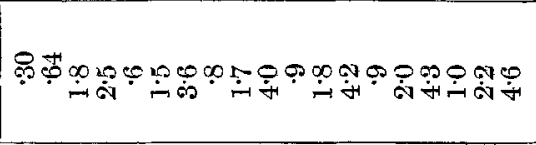 \\
\hline 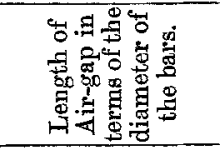 & 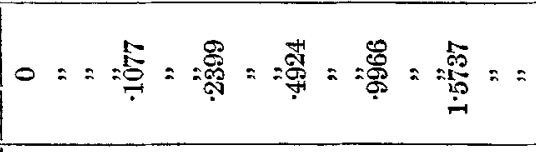 \\
\hline 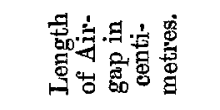 & 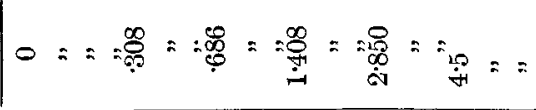 \\
\hline
\end{tabular}


108 Prof. R. Threlfall on an Approximate Method

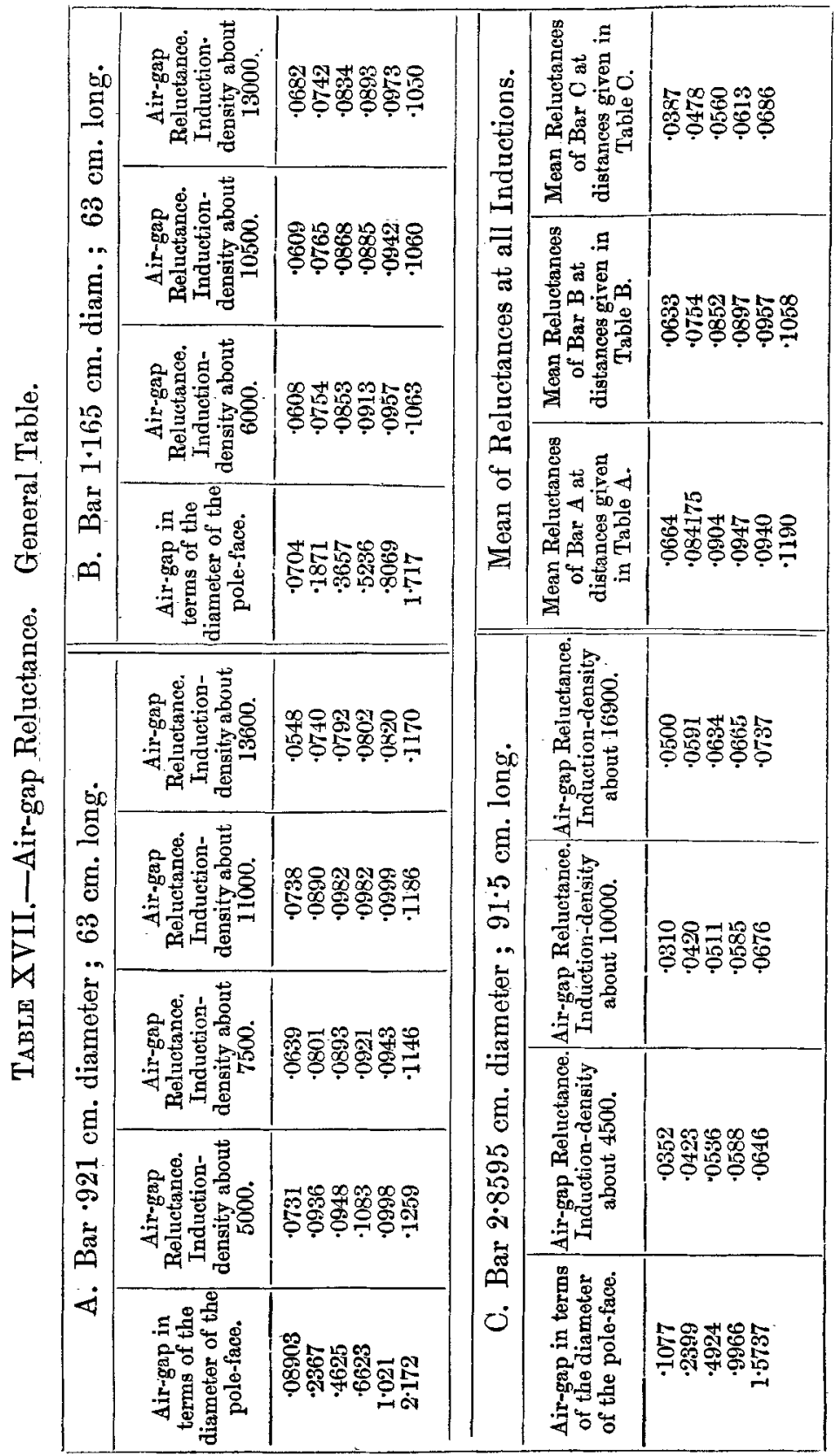


of finding the Forces acting in Magnetic Circuits. 109

Fig. 2.

Reluctance of Air-gaps.

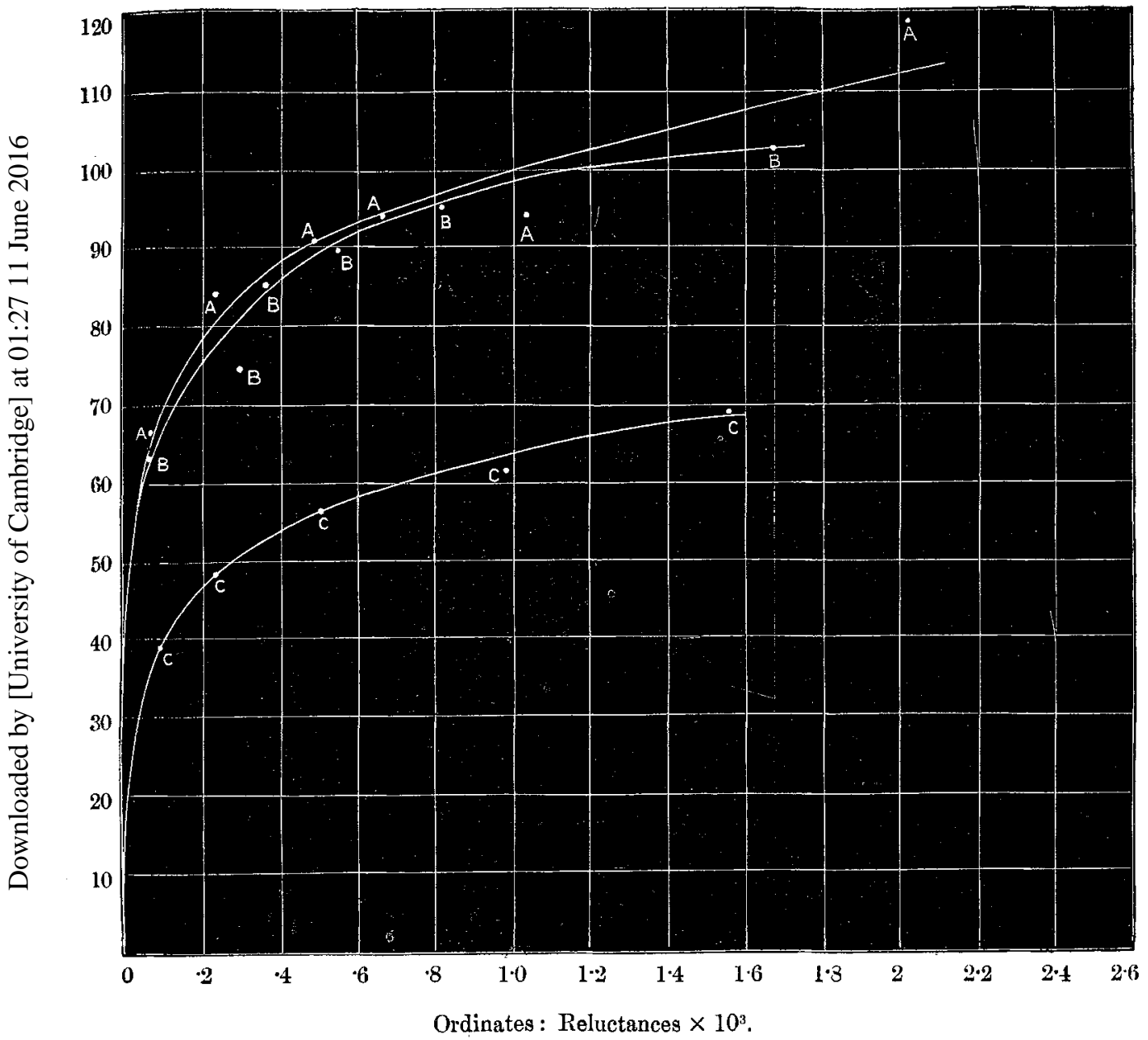

Abscisse : Air-gaps in diameters.

Lettering as in General Table XVII. 
$\S 19$. We can make a comparison between the work done by a ring magnet when it is divided at one point with the work done when the ring is divided at two points. The reluctance data show that though the mean air-gap reluctance may be larger than that of the iron, it is not very greatly so in any practical case, and we can therefore obtain no information by supposing that one is much greater or less than the other; but must proceed by actual trial from the curves to find out which is the most efficient arrangement.

$\$ 20$. In the case of a mechanism represented by a ring divided at one point only, we must remember that the closure of the induction curves involves a "sliding" magnetic contact, and if friction on the bearings is to be avoided this practically ties us down to iron of symmetrical form.

\$21. Incidentally $I$ had occasion to observe the change of reluctance caused by cutting a bar, and then grinding and polishing the ends. This was not done quite so well as in our most successful attempts. The reluctance corresponded to a separation of the bars by about 20 wave-lengths of sodium light, but I am certain that the bars could not have been half so far apart as this, so the surface reluctance is still unaccounted for.

Sydney, 13th July, 1893.

VIII. On a new Harmonic Analyser.

By Prof. O. HENRich, F.R.S.*

1. A COORDING to the theory of Fourier's Series any A function $y$ of $x$ can, under certain restrictions, be expanded in a series progressing according to cosines and sines of nimltiples of $x$ :

This function may be represented graphically by a curve, $x$ and $y$ being taken as rectangular co-ordinates, or it may be defined by aid of such a curve.

Anyhow, we shall suppose this curve given, and also that it extends from $x=0$ to $x=c$ (fig. 1). For this interval the curve may be drawn perfectly arbitrary as long as it gives for every $x$ one single finite value of $y$. This implies that if a point moves along the curve the corresponding value of $x$ always increases. The curve may, however, be discontinuous, so that for a particular valtie of $x$ the urdinate changes suddenly from a value $y_{2}$ to a value $y_{2}$, as from $\mathrm{C}$ to $\mathrm{C}^{\prime}$ in

* Conmunicated by the Physical Society: read March 9, 1894. 